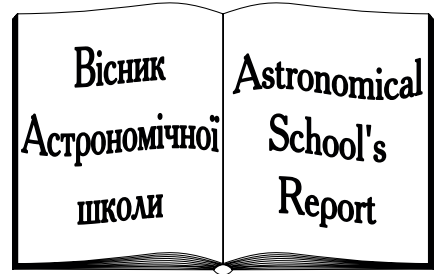

ISSN 2411-6602 (Online)

ISSN 1607-2855 (Print)

Volume $11 \bullet$ Issue $2 \bullet 2015 \quad$ P. $122-126$

UDC 523.4

\title{
A comparative analysis of the geometric and dynamic shapes of the planetary satellites
}

\author{
S.A. Yasenev \\ National Aviation University
}

Determining of dynamic shapes of planetary satellites is today the urgent problem. The purpose of this paper is to analyze the physical properties of satellites which we refer to planetoids.Planetary satellites are analyzed as self-gravitating structures, and differences in their dynamic and geometric shapes are identified.

ПОРІВНЯЛЬНИЙ АНАЛІЗ ГЕОМЕТРИЧНИХ І ДИНАМІЧНИХ ФІГУР СУПУТНИКІВ ПЛАНЕТ, Ясенев С.О. - На сьогоднішній день актуально стае проблема визначення динамічних фігур супутників планет. Мета даної статті полягає у аналізі фізичних властивостей супутників, які ми відносимо до планетоїдів. Проводиться аналіз супутників планет як самогравітуючих утворень $і$ визначаються відмінності ї динамічних $i$ геометричних фігур.

СРАВНИТЕЛЬНЫЙ АНАЛИЗ ГЕОМЕТРИЧЕСКИХ И ДИНАМИЧЕСКИХ ФИГУР СПУТНИКОВ ПЛАНЕТ, ЯСенев С.A. - На сегодняшний день актуальной становится проблема определения динамических фигур спутников планет. Цель данной статьи состоит в анализе физических свойств спутников, которые мы относим к планетоидам. Проводится анализ спутников планет как самогравитирующих образований и определяются различия их динамических и геометрических фигур.

Ключевые слова: спутники планет; фигура; гравитационное поле; форма; масса.

Key words: moons of the planets; figure; gravitational field; form; mass.

\section{INTRODUCTION}

Current astronomical observations carried out with the help of the ground and space means, led to the discovery of a large number of planetary satellites - more than 180, and their number is increasing [5].

In the solar system there are massive satellites that keep their spherical shape by their own gravity, so that is in their evolution gravitational field and proper rotation play a key role. We are interested in dynamic shape of planetary satellites, which we can designate as "planetoids", the self-gravitating entities. By their own weight and its distribution, and gravitational power they hold their shape and strive, to keep it closer to equilibrium. This demands a thorough and detailed study of their space and mechanical properties $[2,4]$.

When considering the companion planet, its free surface in general is not the only object of study. In the study of planetary satellites figures as self-gravitating structures, it is usually assumed that the density is a function of increasing distance to the surface of the body. An individual satellite planet can be studied as spatial, self-gravitating object that provides motion in the gravitational field of the planet. One of level surfaces of gravity usually means the shape, and the body that is limited by the level surface, is called a "planetoid". However, all satellites are significantly influenced by their planet and this cannot be neglected.

\section{ANALYSIS OF RESEARCHES AND PUBLICATIONS}

A large number of planetary satellites have significant compression due to gravitational interaction with their planets, so their gravitational potential is better described as ellipsoid. However, at the moment there are a of problems related to establishment and operation of mathematical models of dynamic figures and gravitational fields $[6,7,10]$. When modeling the gravitational field, scientists use various methods such as Laplace expansion in number, expanding by Lame function, method of point masses; equigravitational figures and other methods are also used $[6,9,11]$.

As for the theory of figures in this direction, the most important methods were proposed by P. Laplace, J. Lagrange, C. Maclaurin, K. Jacobi, P. Pitsetti, A. Clairaut, A. Legendre, K. Gauss, A. Lyapunov, A. Poincare, N. Subbotin, D. Darwin, D. Jeans, J. Lowville and others. There are scientific researches in these issues made by such scholars as K. Holshevnikov, A. Orlov, V. Panteleev, L. Lukyanov, H. Shyrmin, N. Pytyev, V. Titov, I. Nikiforov, A. Martynov, A. Rubinov, L. Sokolov, V. Antonov, O. Zheleznyak, B. Kondratyev, D. Uchayev, I. Prutov and others.

Yasenev Sergey Alexandrovich; $\square$ yasenev91@gmail.com 


\section{STATEMENT OF THE MAIN MATERIAL}

Let us assume that planetary satellites parameters will exceed the critical value for self-gravitating structures [1] and as satellites of the planets rotate around their axes, their forms are not an exact sphere and have some polar compression, that is equatorial radius $R_{\mathrm{eq}}$ will be more polar $R_{\mathrm{pol}}$ [8].

Let's consider that the satellite is a nonchemical solid body moving around the planet. The planet is a gravitational point. The companion form is described as a triaxial ellipsoid of inertia with major semi-axis $a>b>c$ and the main central moments of inertia $A<B<C$.

The stable figure of a celestial body has the property of being perpendicular to the vector sum of the gravitational and centrifugal acceleration. The ratio of these values is as follows:

$$
\frac{\omega^{2} R}{g}=\frac{\omega^{2} R^{3}}{m G}
$$

where $\omega$ is angular velocity being, the main parameter which determines the shape of the satellite. The eccentricity of the satellite is close to the value of the ratio of centrifugal and gravitational acceleration (see Table 1):

$$
\frac{R_{\mathrm{eq}}-R_{\mathrm{pol}}}{R_{\mathrm{eq}}} \approx \frac{\omega^{2} R^{3}}{m G} .
$$

As we can see from Table 1, geometric compression of satellites planets figures do not meet their dynamic compression; this indicates that the satellite has additional effect. Obviously, the biggest impact on the shape of the satellite will be the impact by its planet. Let's consider this in detail. Let's find $\Phi$ is the potential of all forces acting on the satellite that is constant surface potential with constant pressure and density. Since this surface is a surface of constant potential: $\Phi=$ const.

Table 1. Geometric and dynamic compression of planets satellites.

\begin{tabular}{|c|c|c|c|c|c|c|c|}
\hline Name & Mass, $10^{20} \mathrm{~kg}$ & $R_{\mathrm{eq}}, \mathrm{km}$ & $R_{\mathrm{pol}}, \mathrm{km}$ & $\frac{R_{\mathrm{eq}}-R_{\mathrm{pol}}}{R_{\mathrm{eq}}}$ & $R, \mathrm{~km}$ & $P, \mathrm{~s}$ & $\frac{\omega^{2} R^{3}}{m G}$ \\
\hline Ganymede & 1481,90 & 2634,40 & 2633,80 & 0,0002 & 2634,10 & 616503,42 & 0,00019 \\
Titan & 1345,20 & 2578,00 & 2574,00 & 0,0016 & 2576,00 & 1374315,80 & 0,00004 \\
Callisto & 1077,00 & 2411,80 & 2408,80 & 0,0012 & 2410,30 & 1438077,16 & 0,00004 \\
Io & 1893,20 & 1830,00 & 1815,30 & 0,0080 & 1821,00 & 152424,12 & 0,00172 \\
Moon & 734,80 & 1738,14 & 1735,97 & 0,0012 & 1737,10 & 2354086,64 & 0,000008 \\
Europe & 480,20 & 1561,40 & 1560,20 & 0,0008 & 1561,00 & 305968,36 & 0,00050 \\
Triton & 214,00 & 1354,50 & 1352,50 & 0,0015 & 1354,00 & 506385,83 & 0,00027 \\
Titania & 35,27 & 790,00 & 786,80 & 0,0041 & 788,50 & 750143,78 & 0,00015 \\
Oberon & 30,14 & 764,00 & 758,80 & 0,0068 & 761,50 & 1159767,44 & 0,00006 \\
Rhea & 23,07 & 766,20 & 762,20 & 0,0052 & 764,50 & 389288,95 & 0,00076 \\
Iapetus & 18,06 & 747,40 & 712,40 & 0,0468 & 735,80 & 6835390,12 & 0,000003 \\
Charon & 15,80 & 614,00 & 598,00 & 0,0261 & 606,00 & 550329,47 & 0,00028 \\
Ariel & 13,50 & 581,10 & 577,50 & 0,0062 & 578,90 & 217133,28 & 0,00180 \\
Umbriel & 11,75 & 586,10 & 583,30 & 0,0048 & 584,30 & 357063,62 & 0,00079 \\
Dione & 10,96 & 564,40 & 559,60 & 0,0085 & 562,00 & 235830,87 & 0,00172 \\
Tethys & 6,18 & 538,40 & 526,30 & 0,0225 & 531,20 & 162677,63 & 0,00542 \\
Enceladus & 1,10 & 256,60 & 248,30 & 0,0323 & 252,20 & 118044,68 & 0,00619 \\
Miranda & 0,71 & 240,40 & 232,90 & 0,0312 & 236,50 & 121749,73 & 0,00744 \\
\hline
\end{tabular}

Let's consider now the companion planet. Let's assume that its shape is in equilibrium and the planet is in its equatorial plane that we take the point on the body. In this case:

$$
\Phi=\Phi_{S}+\Phi_{P}+\Phi_{C}=\text { const, }
$$

where $\Phi_{S}-$ gravitational potential of the satellite, $\Phi_{P}-$ potential gravity of the planet, $\Phi_{C}-$ centrifugal potential due to of the satellite's own rotation.

The gravitational potential of the satellite:

$$
\Phi_{S}=\frac{G M_{S}}{r}\left[1+\sum_{n=2}^{\infty} \sum_{m=0}^{\infty} \frac{3}{2 n+1} \frac{\sigma_{S}}{\sigma_{m}}\left(\frac{r_{0}}{r}\right)^{n} \times\left(a_{n m} \cos m \lambda+b_{n m} \sin m \lambda\right) P_{n m} \cdot \sin \varphi\right],
$$

where $G$ - the gravitational constant, $M_{S}$ - mass of the satellite, $r$ - radius vector of the satellite shapes, $n$ and $m$ - order and degree with coefficients of spherical functions, $r_{0}$ - radius of the sphere of relativity, $a_{n m}$ and $b_{n m}$ - harmonic coefficients, and $\varphi$ and $\lambda$ - longitude and latitude of the satellite, $\sigma_{S}-$ density of relief, $\sigma_{m}-$ average density of the satellite.

The gravitational potential of the planet:

$$
\Phi_{P}=\frac{G M_{P}}{r_{P}} \frac{G M_{P}}{\sqrt{(\Delta-x)^{2}+y^{2}+z^{2}}}=G M_{P} \cdot\left(\Delta \sqrt{1-\frac{2 x}{\Delta}+\frac{x^{2}}{\Delta^{2}}+\frac{y^{2}}{\Delta^{2}}+\frac{z^{2}}{\Delta^{2}}}\right)^{-1},
$$


where $G$ - the gravitational constant, $M_{P}-$ mass of the planet, $r_{P}$ - distance from the satellite to the centre of the planet, $\Delta$ - distance between the centers of the satellite and planet. In this condition the axis $z$ coincides with the axis of rotation of the satellite and the axis $x$ is directed to the planet, and the axis $y$ is in the direction of motion.

By expanding $1 / r_{P}$ in a series of degrees $\frac{x}{\Delta}, \frac{y}{\Delta}, \frac{z}{\Delta}$ and holding members of the second order of smallness, we obtain:

$$
\Phi_{P}=\frac{G M_{P}}{\Delta}\left(1+\frac{x}{\Delta}+\frac{2 x^{2}-y^{2}-z}{2 \Delta^{2}}\right)
$$

Let us find the potential of the centrifugal force. Let the moon rotates on its axis, which is perpendicular to the orbital plane with a constant angular velocity $\omega$, which is equal to the speed of its orbital motion $\Omega$, that is $\omega=\Omega$. Then the motion of the satellite is a rotation with angular velocity $\omega$ around the axis that runs through the centre of mass of the system "satellite-planet" and which departs from the centre of the satellite at the distance $\Delta \cdot M_{P} /\left(M_{S}+M_{P}\right)$.

The potential of the centrifugal force will be the following:

$$
\Phi_{C}=\frac{1}{2} \omega^{2}\left[\left(\frac{\Delta}{1+\left(M_{S} / M_{P}\right)}-x\right)^{2}+y^{2}\right] .
$$

We perform replacement of $\omega^{2}$ with its expression through the Kepler's third law: $\omega^{2}=G M_{P}(1+\mu) / \Delta^{3}$, where $\mu=M_{S} / M_{P}$.

Then:

$$
\Phi_{C}=\frac{G M_{P}}{\Delta}\left[\frac{1}{2(1+\mu)}-\frac{x}{\Delta}+\frac{x^{2}+y^{2}}{2 \Delta^{2}}\right]
$$

When adding $\Phi_{P}$ and $\Phi_{C}$, we obtain:

$$
\Phi_{P}+\Phi_{C}=\frac{G M_{P}}{\Delta}\left(1+\frac{1}{2(1+\mu)}\right)+\frac{G M_{P}}{2 \Delta^{3}}\left(3 x^{2}-z^{2}\right)+\mu \frac{G M_{P}}{2 \Delta^{3}}\left(x^{2}+y^{2}\right)
$$

The sum (9) is a disturbing potential, which determines the deviation surface (figure) of the satellite from the sphere.

We add to (9) member $\lambda\left(x^{2}+y^{2}+z^{2}\right)$, which by virtue of symmetry may cause slight overall expansion of the satellite, but not significantly affecting its shape. The parameter $\lambda$ is selected so that perturbing potential can be expressed as second order harmonic functions: $\nabla^{2}\left(\Phi_{P}+\Phi_{C}+\lambda r^{2}\right)=0$.

Performing differentiation, we obtain:

Now the disturbing potential is represented as:

$$
\frac{2 G M_{P}}{\Delta^{3}}+6 \lambda=0, \quad \text { where } \quad \lambda=-\frac{G M_{P}}{3 \Delta^{3}} .
$$

$$
\Phi_{\text {gen }}=\Phi_{P}+\Phi_{C}+\lambda r^{2}=\frac{G M_{P}}{\Delta}\left(1+\frac{1}{2(1+\mu)}\right)-\frac{G M_{P}}{3 \Delta^{3}} r^{2}+\mu \frac{G M_{P}}{2 \Delta^{3}}\left(x^{2}+y^{2}\right)+\frac{G M_{P}}{2 \Delta^{3}}\left(3 x^{2}-z^{2}\right),
$$

or bearing in mind that both the first term (constant), and the third one (small) can be rejected, we write (10) as:

$$
\Phi_{\text {gen }}=\frac{G M_{P}}{2 \Delta^{3}}\left(3 x^{2}-z^{2}\right)-\frac{G M_{P}}{3 \Delta^{3}} r^{2}=\frac{G M_{P}}{6 \Delta^{3}}\left(7 x^{2}-2 y^{2}-5 z^{2}\right) .
$$

The surface of the satellite is acted upon by the disturbing potential and becomes deformed. Deformation is expressed by harmonic function of the second order $J_{2}$ :

$$
r=r_{0}\left(1+\varepsilon J_{2}\right)
$$

where $r$ - radius vector shapes of the satellite, $r_{0}$ - radius of the sphere of relativity, $\varepsilon-$ ratio $\sigma_{m} / \sigma_{S}\left(\sigma_{m}-\right.$ average density of the satellite, $\sigma_{S}$ - density of relief), $J_{2}$ - dynamic parameter that characterizes flattening figure of the satellite.

Confining harmonic functions of the second order, we obtain from (1) the potential of a homogeneous spheroid:

$$
\Phi_{S}=\frac{G M_{S}}{r}\left(1+\frac{3}{5} \frac{\varepsilon J_{2} r_{0}^{2}}{r^{5}}\right)
$$

We introduce (12) into the expression for the potential spheroid (13), then:

$$
\Phi_{S}=\frac{G M_{S}}{r_{0}\left(1+\varepsilon J_{2}\right)}\left[1+\frac{3}{5} \frac{\varepsilon J_{2}}{\left(1+\varepsilon J_{2}\right)^{2}}\right]=\frac{G M_{S}}{r_{0}}\left[\left(1-\varepsilon J_{2}\right)\left(1+\frac{3}{5} \frac{\varepsilon J_{2} r_{0}^{2}}{r_{0}\left(1+\varepsilon J_{2}\right)^{2}}\right)\right] .
$$

When adding capacity satellite (14) and the disturbing potential (11), we obtain the equation of the tiered moon's surface:

$$
\Phi=\frac{G M_{P}}{6 \Delta^{3}}\left(7 x^{2}-2 y^{2}-5 z^{2}\right)+\frac{G M_{S}}{r_{0}} \times\left[\left(1-\varepsilon J_{2}\right)\left(1+\frac{3}{5} \frac{\varepsilon J_{2} r_{0}^{2}}{r_{0}\left(1+\varepsilon J_{2}\right)^{2}}\right)\right]=\text { const. }
$$




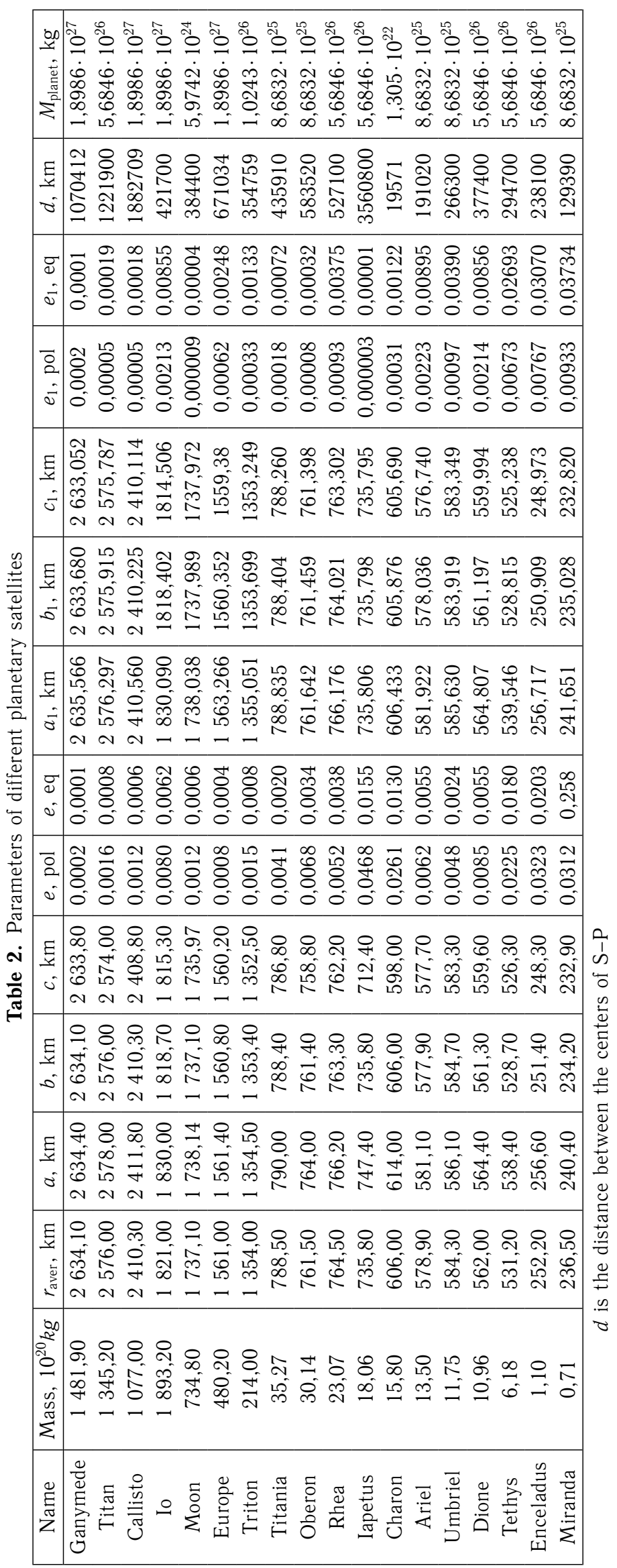


Neglecting the small second order and assuming constant equal $G M_{S} / r_{0}$, we determine from (15) the value $\varepsilon J_{2}$ :

$$
\varepsilon J_{2}=\frac{5}{12} \frac{M_{P}}{M_{S}} \frac{r_{0}^{3}}{\Delta^{3}} \frac{\left(7 x^{2}-2 y^{2}-5 z^{2}\right)}{r_{0}^{2}} .
$$

Let us enter the value in (12). Now equation of the tiered moon's surface up to the second harmonic order will look like:

$$
r=r_{0}\left(1+\frac{5}{12} \frac{M_{P}}{M_{S}} \frac{r_{0}^{3}}{\Delta^{3}} \frac{\left(7 x^{2}-2 y^{2}-5 z^{2}\right)}{r_{0}^{2}}\right) .
$$

Consistently stating in (17) that $x=r_{0}, y=r_{0}, z=r_{0}$ let us find semiaxes of the satelloid (planetoid) [3]:

$$
\left\{\begin{array}{l}
a=r_{0}\left(1+\frac{35}{12} \frac{M_{P}}{M_{S}} \frac{r_{0}^{3}}{\Delta^{3}}\right), \\
b=r_{0}\left(1-\frac{10}{12} \frac{M_{P}}{M_{S}} \frac{r_{0}^{3}}{\Delta^{3}}\right), \\
c=r_{0}\left(1-\frac{25}{12} \frac{M_{P}}{M_{S}} \frac{r_{0}^{3}}{\Delta^{3}}\right) .
\end{array}\right.
$$

It follows that the largest satellite axis is the axis directed to the planet, the second largest axis is that which is directed toward the orbital motion of the satellite (on the same surface of the first one and perpendicular to it), and the third axis between the poles is the smallest.

Substituting the values $r_{0}, M_{P}, M_{S}, \Delta$, we obtain numerical values of semiaxes, polar and equatorial compression of planetoids (see Table 2).

These values do not coincide with the results of measurements, indicating the unequal distribution of density in planetary satellites. That is the figures of planetary satellites differ from hydrostatic equilibrium shapes.

For some simple models with the help of mathematical analysis methods there have been already found correlations between the degree of attending at the poles and parameters, characterizing the density distribution [7]. For example, the rotational body with uniform density with one axis of symmetry passing through the poles, known as Maclaurin spheroids, rotating bodies with mass concentrated at a single point (in the centre of the body) are variations of Roche models. All existing forms of planetary satellites are between these two options.

\section{CONCLUSIONS}

1. It was found that the geometric shape of planetary satellites compression is out of accord with their dynamic compression, indicating uniform density distribution inside the satellite.

2. Due to the planet's gravitational interaction with the satellite there arise tidal forces that distort the dynamic equilibrium shape of the satellite and the satellite forms contribute to the emergence of a triaxial ellipsoid whose axis is the largest oriented toward the planet, the second largest one in the direction of orbital motion, the third one - between the poles.

3. A comparison of dynamic and geometric characteristics of planetary satellites gave us an idea of potential deformation of shapes. We can use these data to get an idea of the distribution of the matter inside the satellites and thus the knowledge of their internal structure.

1. Byalko A.V., M.: Sci., 1989. - 240 p.

2. Vidmachenko A.P., Morozhenko O.V. // Profi, 2014. - 388 p.

3. Grushinskiy N.P., M.: Sci., 1976. - 512 p.

4. Emelyanov N.V., Uralskaya V.S. // Astron. report. - 2011. - 45(5). - P.387-395.

5. Natural satellites of the planets. - http://Infm1.sai.msu.ru/neb/rw/natsat.

6. Zavizion O.V. // KAMMAK-99. - Vinnytsya, 2000. - P.409-417.

7. Kondratyev B.P. // Udmurt univer. report. - 2012. - 2. - P.11-30.

8. Kulikov K.A., Sidorenko N.S., M.: Sci., 1977. - 191 p.

9. Malinnikov V.A., Oberst Y., Uchaev D.V., Prutov I.S. // Geod. Aer. Photogr. - 2011. - 6. - P.64-68.

10. Murray K., Dermott C., M.: Fizmatlit, 2010. - 588 p.

11. Uralskaya V.S. // Astron. report. - 2003. - 37 (5). - P.371-399.

Received 8.10.2015

Accepted 13.11.2015 